\title{
Analysis of 3D Cobweb Economic Differential Dynamic System Based on Supply-Demand and Price Relationships
}

\author{
Debao Gao \\ College of Science, Heilongjiang Bayi Agricultural University, Daqing, Heilongjiang 163319, China \\ Correspondence should be addressed to Debao Gao; godebao@byau.edu.cn
}

Received 31 October 2021; Accepted 5 February 2022; Published 7 March 2022

Academic Editor: Mustafa R. S. Kulenovic

Copyright $\odot 2022$ Debao Gao. This is an open access article distributed under the Creative Commons Attribution License, which permits unrestricted use, distribution, and reproduction in any medium, provided the original work is properly cited.

\begin{abstract}
When the supply and demand of commodities are out of equilibrium, the dynamic changes in supply and demand are mainly manifested in the dynamic fluctuation of commodity prices and the change of price will drive the change of supply and demand. This paper proposes a method for constructing a three-dimensional cobweb economic model using the "predator-prey" theory. Firstly, based on the intrinsic driving relationships among supply, price, and demand, a differential dynamic model of the commodity and a pulse differential model when productivity increases are established. Secondly, the differential dynamics theory is used to analyze the existence of the model's positive equilibrium and to prove its global asymptotic stability, uniform boundedness, persistence, and existence of the periodic solution. Finally, the correctness of the conclusions of the model is verified by numerical simulation. The relevant conclusions of model (1) reveal the evolution laws of supply, price, and demand that fluctuate around the positive equilibrium and tend to balance. Model (32) reveals that with the continuous improvement of production technology, supply quantity, price, and demand will fluctuate, but all three will change periodically. These conclusions can provide valuable help for people in the production, sale, and purchase of goods.
\end{abstract}

\section{Introduction}

It has always been an important subject for scholars and businessmen to explore the dynamic operation law of the commodity economy. Because it is very difficult to do experimental research on economic operation law, the mathematical model has become an important tool to analyze economic operation law. For example, the statistical model [1] is an indispensable mathematical tool in the econometric theory [2]. The separation theorem [3] of the investment theory is expressed by the probability model and the optimization model [4]. The application and research of mathematical model in economics have many specific elaborations in [5], which will not be repeated here.

The demand, supply, and price are essential elements in all commodity economy systems. The cobweb economy models [6-9] are a mathematical system to study the relationships among them. Most cobweb economic systems only study the law of commodity operation from the external relationship between demand and price or between supply and price. Few scholars set out to construct a mathematical system from the internal driving force between them to explore the relationships among demand, price, and supply.

Demand is the driving force of the commodity economy. Without demand, there will be no supply. The purpose of supply is to maximize the utility of goods. The marginal utility of goods [10] determines the price, and the total utility determines the supply of goods. When the quantity of supply changes, it will drive the change of price, and the change of price will drive the change of demand. Conversely, when demand changes, it also drives price and supply changes. The external relations among them are as follows: when the quantity of goods exceeds the demand, the price will decrease; when supply is less than demand, the price will rise. The change of commodity quantity or price is a process changing with time. When the price rises, it will drive the increase of commodity quantity, and the increase of commodity quantity will hinder the rise of price. Generally, the change of price will also affect the demand: when the price 
increases, the demand will decrease. When the price decreases, the demand will increase moderately.

According to the above analysis, the relationship between supply and price or between price and demand is the relationship between predator and prey [11-14] in biomathematics [15]. More specifically, prices exist for predator demand and supplies prey on price. The quantity of supply (the number of predators) increases (or decreases) with the increase (or decrease) of the price (the number of prey). In this paper, the differential dynamics theory between predator and prey is applied to construct the differential dynamic system among supply, price, or demand and then to explore the dynamic operation law among them.

By revealing the internal driving relationship among supply, price, and demand, it can help people predict the development trend of supply and demand scientifically and provide the basis for making stable price policy. Businesses can adjust production, circulation, or procurement to effectively avoid risks according to the development trend of supply and demand. Under the condition of ensuring that the price does not fluctuate obviously, using the development trend of supply and demand to promote the flexible change of price can create a benign business competition environment.

\section{Model Construction and Analysis}

The price and supply of goods are based on the demand of consumers. Many commodities, such as pork, eggs, or seafood, will decrease in demand when prices rise. When the price decreases, the demand will increase. Under the condition that other conditions remain unchanged, the demand and price of goods change in the opposite direction. Because the number of consumers is limited, there is an upper limit on demand. The supply of goods also varies with the price. When the price rises, the supply will increase. When the price decreases, the supply will decrease. In addition, when the supply increases to "oversupply" with the increase of price, the goods will be sold at a lower price, resulting in some decreases in the price of goods. The decline in commodity prices will stimulate the increase in demand. When the demand increases to "demand exceeds supply," commodity prices will rise again, leading to an increase in supply.

Before establishing the differential dynamics model of the relationship among demand, price, and supply, some following assumptions need to be given about the model.

(1) Limited by consumption capacity, this article assumes that the demand has an upper limit, and in other words, the demand is density dependent [16-18].

(2) The increase in demand has the characteristics of rapid and direct functional response to price, and it can be assumed that the functional response is the linear relation.
(3) Price is the external manifestation of the game between demand and supply. It can be assumed that the change rate of price is inversely proportional to supply and directly proportional to demand.

(4) Because most commodities require production time, the increase in supply does not often have a linear relationship with the functional response of price. Assume that the functional response is a type II functional response [17-19].

Let $D(t), P(t)$, and $S(t)$ be the dimensionless functions of demand, price, and supply of goods at time $t$, respectively. Based on the above analysis and assumptions, the threedimensional cobweb economic differential dynamics system based on the relationship among supply, price, and demand is as follows.

$$
\left\{\begin{array}{l}
\frac{\mathrm{d} D}{\mathrm{~d} t}=D\left(e_{1}-k D-\alpha P\right) \\
\frac{\mathrm{d} P}{\mathrm{~d} t}=P\left(-e_{2}+\beta D-\gamma S\right) \\
\frac{\mathrm{d} S}{\mathrm{~d} t}=S\left(-e_{3}+\frac{\delta P}{\omega+P}\right)
\end{array}\right.
$$

where $e_{1}, e_{2}, e_{3}, k, \alpha, \beta, \gamma, \delta, \omega$ are all positive real numbers. Their specific economic meanings are shown in Table 1.

The initial conditions of system (1) are given as

$$
D(0)>0, P(0)>0, S(0)>0 \text {. }
$$

Known from the practical applicability of system (1), its feasible domain is

$$
R_{+}^{3}=\left\{(D, P, S) \in R^{3}: D \geq 0, P \geq 0, S \geq 0\right\} .
$$

It is easy to know that the right end of system (1) is smooth and continuous in $R_{+}^{3}$. Therefore, system (1) has a unique solution in $R_{+}^{3}$. System (1) fully considers the functional response of demand, price, supply, and their mutual interference, expresses the differential dynamic relationships among demand, price, and supply, and has practical significance and theoretical value. Based on the economic significance, this article only conducts a qualitative analysis of system (1) in $R_{+}^{3}$.

\subsection{Property Analysis of System (1)}

Theorem 1. All solutions of system (1) with the initial conditions (2) are positive for all $t \geq 0$.

Proof. It is easy to know that system (1) is equivalent to the following system: 
TABLE 1: Economic meaning of parameters.

\begin{tabular}{lc}
\hline Parameter & Economic meaning \\
\hline$e_{1}$ & The intrinsic growth rate of the demand \\
$e_{2}$ & The consumption rate after price preys on demand \\
$e_{3}$ & The consumption rate after supply preys on price \\
$k$ & The environmental density restriction coefficient of \\
$\alpha$ & demand \\
$\beta$ & The predation rate of price to demand \\
$\gamma$ & The conversion rates after price-predation demand \\
$\delta$ & The conversion rates after supply-predation price \\
\hline
\end{tabular}

$$
\left\{\begin{array}{l}
D(t)=D(0) \exp \left\{\int_{0}^{t}\left[e_{1}-k D(u)-\alpha P(u)\right] \mathrm{d} u\right\}, \\
P(t)=P(0) \exp \left\{\int_{0}^{t}\left[-e_{2}+\beta D(u)-\gamma S(u)\right] \mathrm{d} u\right\}, \\
S(t)=S(0) \exp \left\{\int_{0}^{t}\left[-e_{3}+\frac{\delta P(u)}{\omega+P(u)}\right] \mathrm{d} u\right\} .
\end{array}\right.
$$

It can be seen if $D(0)>0, P(0)>0, S(0)>0$, there will always be $D(t)>0, P(t)>0, S(t)>0$ for all $t>0$. This ends the proof of Theorem 1 .

In practice, the price, demand, and supply of commodities hardly have negative values, so the conclusion of Theorem 1 is true.

Lemma 1 (see [20]). Consider the scalar differential equation

$$
\frac{\mathrm{d} u}{\mathrm{~d} t}=f(t, u), u\left(t_{0}\right)=u_{0}
$$

where $f(t, u)$ is continuous in $t$ and locally Lipschitz in $u$, for all $t \geq 0$ and all $u \in J s \subset R$. Let $\left[t_{0}, T\right)$ (T could be infinity) be the maximal interval of existence of the solution $u(t)$, and suppose $u(t) \in J$ for all $t \in\left[t_{0}, T\right)$. Let $v(t)$ be a continuous function whose upper right-hand derivative $D^{+} v(t)$ satisfies the differential inequality

$$
D^{+} v(t) \leq f(t, v(t)), v\left(t_{0}\right) \leq u_{0}
$$

with $v(t) \in J$ for all $t \in\left[t_{0}, T\right)$. Then, $v(t) \leq u(t)$ for all $t \in\left[t_{0}, T\right)$.

Lemma 1 is called the comparison principle.

Theorem 2. If $\omega-1 \geq 0$, all positive solutions of system (1) are uniformly bounded [18] in $R_{+}^{3}$.

Proof. Define a function

$$
V(D, P, S)=\beta D+\alpha P+\frac{\alpha \gamma}{\delta} S .
$$

Then,

$$
\begin{aligned}
\frac{\mathrm{d} V}{\mathrm{~d} t} & =\beta \frac{\mathrm{d} D}{\mathrm{~d} t}+\alpha \frac{\mathrm{d} P}{\mathrm{~d} t}+\frac{\alpha \gamma}{\delta} \frac{\mathrm{d} S}{\mathrm{~d} t} \\
& =\beta\left(e_{1} D-k D^{2}\right)-\alpha e_{2} P-\frac{\alpha \gamma}{\delta} e_{3} S-\alpha \gamma \frac{\omega-1+P}{\omega+P} P S .
\end{aligned}
$$

If $\omega-1 \geq 0$

$$
\frac{\mathrm{d} V}{\mathrm{~d} t}<\beta\left(e_{1} D-k D^{2}\right)
$$

According to Lemma 1, it can be seen that

$$
\lim _{t \longrightarrow+\infty} V(t) \leq \frac{e_{1}}{k} \text {. }
$$

Therefore, the conclusion of Theorem 2 holds. The proof is finished.

In practice, limited by production capacity, the supply is bound to have an upper limit. When the price of a product is higher than the consumer's ability to bear, the consumer will not be able to buy the product, so the price must be within the range of consumers' acceptance. Limited by the number of consumers, the demand must be limited. The results are basically consistent with those of Theorem 2 .

\section{Theorem 3}

(1) System (1) has two boundary equilibriums $E_{0}(0,0,0), E_{1}\left(e_{1} / k, 0,0\right)$ in $R_{+}^{3}$.

(2) If the assumption $H_{1}: \beta e_{1}-k e_{2}>0$ holds, then system (1) has a non-negative equilibrium $E_{2}\left(e_{2} / \beta, \beta e_{1}-\right.$ $\left.k e_{2} / \alpha \beta, 0\right)$.

(3) If the assumptions $H_{2}: \delta-e_{3}>0, H_{3}: e_{1}\left(\delta-e_{3}\right)$ $-\alpha \omega e_{3}>0, H_{4}:\left(\beta e_{1}-k e_{2}\right)\left(\delta-e_{3}\right)-\alpha \beta \omega e_{3}>0$ hold, then system (1) has a positive equilibrium $E_{3}\left(D^{*}, P^{*}, S^{*}\right)$, where

$$
\begin{aligned}
D^{*} & =\frac{e_{1}\left(\delta-e_{3}\right)-\alpha \omega e_{3}}{k\left(\delta-e_{3}\right)}, \\
P^{*} & =\frac{\omega e_{3}}{\delta-e_{3}}, \\
S^{*} & =\frac{\left(\beta e_{1}-k e_{2}\right)\left(\delta-e_{3}\right)-\alpha \beta \omega e_{3}}{k \gamma\left(\delta-e_{3}\right)} .
\end{aligned}
$$

Proof. The non-negative equilibriums of system (1) satisfy the following equations:

$$
\left\{\begin{array}{l}
D\left(e_{1}-k D-\alpha P\right)=0 \\
P\left(-e_{2}+\beta D-\gamma S\right)=0 \\
S\left(-e_{3}+\frac{\delta P}{\omega+P}\right)=0
\end{array}\right.
$$

It is easy to find the boundary equilibrium $E_{0}(0,0,0)$ of system (1). 
If $D \neq 0 P=S=0$, another boundary equilibrium $E_{1}\left(e_{1} / k, 0,0\right)$ can also be obtained.

If $D \neq 0, P \neq 0, S=0$ and $\beta e_{1}-k e_{2}>0$, then system (1) has a non-negative equilibrium

$$
E_{2}\left(\frac{e_{2}}{\beta}, \frac{\beta e_{1}-k e_{2}}{\alpha \beta}, 0\right) .
$$

If $D \neq 0, P \neq 0, S \neq 0$, by solving the third equation in the system of equation (12), we can get

$$
P^{*}=\frac{\omega e_{3}}{\delta-e_{3}}
$$

By substituting the above formula into the first equation, we can obtain

$$
D^{*}=\frac{e_{1}\left(\delta-e_{3}\right)-\alpha \omega e_{3}}{k\left(\delta-e_{3}\right)} .
$$

By substituting the above formula into the second equation, then

$$
S^{*}=\frac{\left(\beta e_{1}-k e_{2}\right)\left(\delta-e_{3}\right)-\alpha \beta \omega e_{3}}{k \gamma\left(\delta-e_{3}\right)} .
$$

It is easy to know if $H_{2}-H_{4}$ is established, $D^{*}>0, P^{*}>0, S^{*}>0$. The proof is completed.

The boundary equilibrium $E_{0}$ indicates the state in which goods are eliminated, such as tape recorder, BPS, and so on, consumers have no demand for them and will not buy them, and then there will be no commodity supply. The boundary equilibrium $E_{1}$ indicates that the product is in a state of "selfsufficiency," the product is only used to satisfy the self, the product has no price, and there is no external supply. The non-negative equilibrium $E_{2}$ is also a boundary equilibrium, which is a state of commodity with "price without market": there is demand and there is price, but there is no supply. The only positive equilibrium $E_{3}$ represents the relative equilibrium state when supply and demand play games with each other.

For exploring the stability of system (1) at the above equilibriums, the Jacobi matrix [18] of system (1) needs to be calculated, and its specific expression is

$$
J(D, P, S)=\left[\begin{array}{ccc}
e_{1}-2 k D-\alpha P & -\alpha D & 0 \\
\beta P & -e_{2}+\beta D-\gamma S & -\gamma P \\
0 & \frac{\omega \delta S}{(\omega+P)^{2}} & -e_{3}+\frac{\delta P}{\omega+P}
\end{array}\right] .
$$

\section{Theorem 4}

(1) The boundary equilibrium $E_{0}$ is unstable.

(2) If $\beta e_{1}-k e_{2}<0$, then the boundary equilibrium $E_{1}$ is locally asymptotically stable; otherwise, it is unstable.

(3) If $\delta-e_{3}<0$ and $H_{1}$ hold, then the non-negative equilibrium $E_{2}$ is locally asymptotically stable.

Proof. The following only proves conclusion (3), and (1) and (2) can be proved by the same method. Let $I$ be the identity matrix, and then the expression of the characteristic equation $\left|J\left(E_{2}\right)-\lambda I\right|=0$ is

$$
\begin{aligned}
& \left|\begin{array}{ccc}
-\frac{k e_{2}}{\beta}-\lambda & -\frac{\alpha e_{2}}{\beta} & 0 \\
\frac{\beta e_{1}-k e_{2}}{\alpha} & -\lambda & -\frac{\gamma\left(\beta e_{1}-k e_{2}\right)}{\alpha \beta} \\
0 & 0 & \frac{\left(\delta-e_{3}\right)\left(\beta e_{1}-k e_{2}\right)-\alpha \beta \omega e_{3}}{\alpha \beta \omega+\beta e_{1}-k e_{2}}-\lambda
\end{array}\right| \\
& =\left[\lambda-\frac{\left(\delta-e_{3}\right)\left(\beta e_{1}-k e_{2}\right)-\alpha \beta \omega e_{3}}{\beta e_{1}-k e_{2}+\alpha \beta \omega}\right]\left[\lambda^{2}+\frac{k e_{2}}{\beta} \lambda+\frac{e_{2}\left(\beta e_{1}-k e_{2}\right)}{\beta}\right]=0 \text {. }
\end{aligned}
$$
then,

Let $\lambda_{1}, \lambda_{2}, \lambda_{3}$ be the three roots of the above equation; 


$$
\begin{aligned}
\lambda_{1} & =\frac{\left(\delta-e_{3}\right)\left(\beta e_{1}-k e_{2}\right)-\alpha \beta \omega e_{3}}{\beta e_{1}-k e_{2}+\alpha \beta \omega}, \\
\lambda_{2}+\lambda_{3} & =-\frac{k e_{2}}{\beta}<0, \\
\lambda_{2} \lambda_{3} & =\frac{e_{2}\left(\beta e_{1}-k e_{2}\right)}{\beta}>0 .
\end{aligned}
$$

If $\delta-e_{3}<0$ and $H_{1}: \beta e_{1}-k e_{2}>0$ hold, it can be seen that $\lambda_{1}$ is a negative number, and $\lambda_{2}$ and $\lambda_{3}$ both have negative real parts. Therefore, according to the Hurwitz discriminant $[17,18]$, it can be known that the non-negative equilibrium point $E_{2}$ is locally asymptotically stable. The proof is completed.

The non-negative equilibrium $E_{0}$ is unstable, indicating that the commodity will inevitably be born. In a local area, if the condition $\beta e_{1}-k e_{2}<0$ holds, then the equilibrium state of the self-sufficient economy is $E_{1}$. If the conversion rate of supply-predation price is less than its consumption rate $\left(\delta-e_{3}<0\right)$, the state of price without market can also be stabilized in local areas.

Theorem 5. If assumptions $\mathrm{H}_{2}-\mathrm{H}_{4}$ hold, then the only positive equilibrium $E_{3}$ of system (1) is locally asymptotically stable.

Proof. Because the Jacobi matrix of system (1) at $E_{3}$ is

$$
J\left(D^{*}, P^{*}, S^{*}\right)-\lambda I=\left[\begin{array}{ccc}
-k D^{*}-\lambda & -\alpha D^{*} & 0 \\
\beta P^{*} & -\lambda & -\gamma P^{*} \\
0 & \frac{\omega \delta^{*} S^{*}}{\left(\omega+P^{*}\right)^{2}} & -\lambda
\end{array}\right] \text {, }
$$

the expression of $\left|J\left(D^{*}, P^{*}, S^{*}\right)-\lambda I\right|=0$ is

$$
\lambda^{3}+M_{1} \lambda^{2}+M_{2} \lambda+M_{3}=0
$$

where

$$
\begin{aligned}
& M_{1}=k D^{*}, \\
& M_{2}=\alpha \beta D^{*} P^{*}+\frac{\omega \gamma \delta P^{*} S^{*}}{\left(\omega+P^{*}\right)^{2}}, \\
& M_{3}=\frac{\omega \gamma \delta k D^{*} P^{*} S^{*}}{\left(\omega+P^{*}\right)^{2}} \\
& H_{1}=M_{1}=k D^{*}>0, \\
& H_{2}=\left|\begin{array}{cc}
M_{1} & M_{3} \\
1 & M_{2}
\end{array}\right|=M_{1} M_{2}-M_{3}=k \alpha \beta\left(D^{*}\right)^{2} P^{*}>0, \\
& H_{3}=\left|\begin{array}{ccc}
M_{1} & M_{3} & 0 \\
1 & M_{2} & 0 \\
0 & M_{1} & M_{3}
\end{array}\right|=M_{3} H_{2}>0 .
\end{aligned}
$$

According to Routh-Hurwitz theorem [18], it is known that all roots of the equation $\left|J\left(D^{*}, P^{*}, S^{*}\right)-\lambda I\right|=0$ have negative real parts. Therefore, the only positive equilibrium $E_{3}$ is locally asymptotic stable. The proof is completed.

Theorem 5 gives the conditions $\mathrm{H}_{2}-\mathrm{H}_{4}$ that the only positive equilibrium is locally asymptotically stable. They are also the conditions for the existence of the positive equilibrium.

Lemma 2 (see [21]). Consider a continuously differentiable three-dimensional differential system

$$
\begin{aligned}
\frac{\mathrm{d} D}{\mathrm{~d} t} & =F(D, P, S), \\
\frac{\mathrm{d} P}{\mathrm{~d} t} & =G(D, P, S), \\
\frac{\mathrm{d} S}{\mathrm{~d} t} & =H(D, P, S),
\end{aligned}
$$

defined in some open simply connected subset $\mathcal{U} \subset R^{3}$. Assume that there exists a continuously differentiable $B(D, P, S): \mathcal{U} \longrightarrow R$, such that

$$
\frac{\partial(B F)}{\partial D}+\frac{\partial(B G)}{\partial P}+\frac{\partial(B H)}{\partial S} \geq 0(\text { or } \leq 0),
$$

vanishing only on a set of zero Lebesgue measure. Then, system (23) has no periodic orbits contained in $\mathcal{U}$. This function B is usually called a Dulac function of system (23).

Lemma 2 is called the Bendixson-Dulac theorem.

Theorem 6. If $k-\beta \geq 0$, then system (1) does not have the limit cycle in $R_{+}^{3}$.

Proof. In system (1), let

$$
\left\{\begin{array}{l}
\frac{\mathrm{d} D}{\mathrm{~d} t}=D\left(e_{1}-k D-\alpha P\right)=F \\
\frac{\mathrm{d} P}{\mathrm{~d} t}=P\left(-e_{2}+\beta D-\gamma S\right)=G \\
\frac{\mathrm{d} S}{\mathrm{~d} t}=S\left(-e_{3}+\frac{\delta P}{\omega+P}\right)=H
\end{array}\right.
$$

and take the Dulac function [21]

$$
B(D, P, S)=\frac{\omega+P}{D P S} \text {. }
$$

Then, if $k-\beta \geq 0$,

$$
\begin{aligned}
\frac{\partial(B F)}{\partial D}+\frac{\partial(B G)}{\partial P}+\frac{\partial(B H)}{\partial S}= & -\frac{k-\beta}{S}-\frac{k \omega}{P S}-\frac{e_{2}}{D S}-\frac{\gamma}{D} \\
& <0,(D, P, S) \in R_{+}^{3} .
\end{aligned}
$$

According to Lemma 2, it can be known that system (1) does not have the limit cycle in $R_{+}^{3}$. This ends the proof. 
According to the conclusions of Theorems 4-6, the following theorem can be obtained.

Theorem 7. Let $k-\beta \geq 0$, and there are the following conclusions.

(1) If $\beta e_{1}-k e_{2}<0$, then the boundary equilibrium $E_{1}$ is globally asymptotically stable.

(2) If $\delta-e_{3}<0$ and $H_{1}$ hold, then the non-negative equilibrium point $E_{2}$ is globally asymptotically stable.

(3) If $\mathrm{H}_{2}-\mathrm{H}_{4}$ hold, then the positive equilibrium $\mathrm{E}_{3}$ is globally asymptotically stable.

2.2. Numerical Simulation of System (1). To observe the global asymptotic stability of the equilibriums of system (1), it will take the initial values

$$
\begin{aligned}
& D(0)=1, \\
& P(0)=1.5, \\
& S(0)=1 .
\end{aligned}
$$

If parameters

$$
\begin{aligned}
e_{1} & =2, \\
e_{2} & =0.1, \\
e_{3} & =0.1, \\
k & =5, \\
\alpha & =0.8, \\
\beta & =0.2, \\
\gamma & =0.1, \\
\delta & =1, \\
\omega & =4
\end{aligned}
$$

are taken, it is easy to verify $k-\beta \geq 0, \beta e_{1}-k e_{2}<0$. According to conclusion (1) of Theorem 7, we can get that the equilibrium $E_{1}=(0.4,0,0)$ is globally asymptotically stable. If the parameter group (29) is selected, the numerical simulation of system (1) is shown in Figure 1.

If the supply $S=0$ and the price $P=0$, the demand for self-sufficiency $D=0.4$ can be sustained for a long time.

If parameters

$$
\begin{aligned}
e_{1} & =7, \\
e_{2} & =0.1, \\
e_{3} & =0.2, \\
k & =5, \\
\alpha & =0.8, \\
\beta & =0.2, \\
\gamma & =0.1, \\
\delta & =0.1, \\
\omega & =4
\end{aligned}
$$

are taken, we can verify $\beta e_{1}-k e_{2}>0, k-\beta>0, E_{2}=(0.5$, $5.625,0)$. At this time, the numerical simulation of system (1) is shown in Figure 2. Figure 2 also verifies that the nonnegative equilibrium $E_{2}$ is globally asymptotically stable.

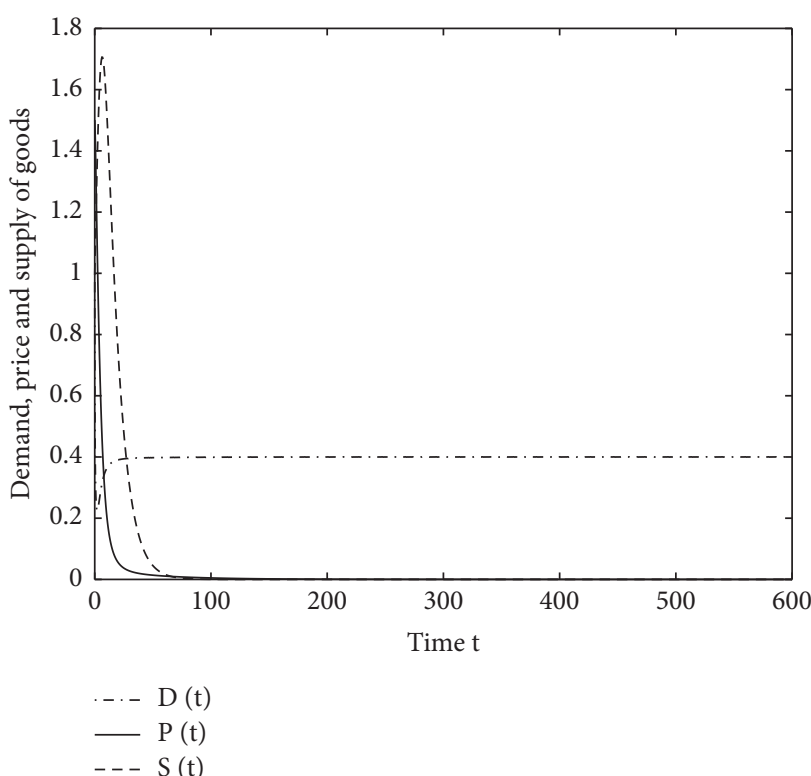

FIgURE 1: Plane phase diagram of the global asymptotic stability at $E_{1}$.

If parameters

$$
\begin{aligned}
e_{1} & =7, \\
e_{2} & =0.1, \\
e_{3} & =0.1, \\
k & =5, \\
\alpha & =0.8, \\
\beta & =0.2, \\
\gamma & =0.1, \\
\delta & =1, \\
\omega & =4
\end{aligned}
$$

are taken, it is easy to verify that the above parameters meet the conditions for the existence of the positive equilibrium and $k-\beta>0$. By calculating, the positive equilibrium $E_{3}=(1.33,0.44,1.66)$. The numerical simulation of system (1) is shown in Figures 3 and 4.

Figures 3 and 4 show that with the passage or extension of time, although the values of demand $D(T)$, price $P(T)$, and supply $S(T)$ will fluctuate continuously, the amplitude will gradually decrease and tend to the positive equilibrium $E_{3}$.

\section{Analysis of the Commodity Dynamic Relationship When Production Technology Is Improved}

With the development of technology and the improvement of production technology, the production cost of goods will inevitably be reduced, and the supply of goods will be temporarily increased, which will lead to the decline of price, and the reduction of price will also promote the temporary 


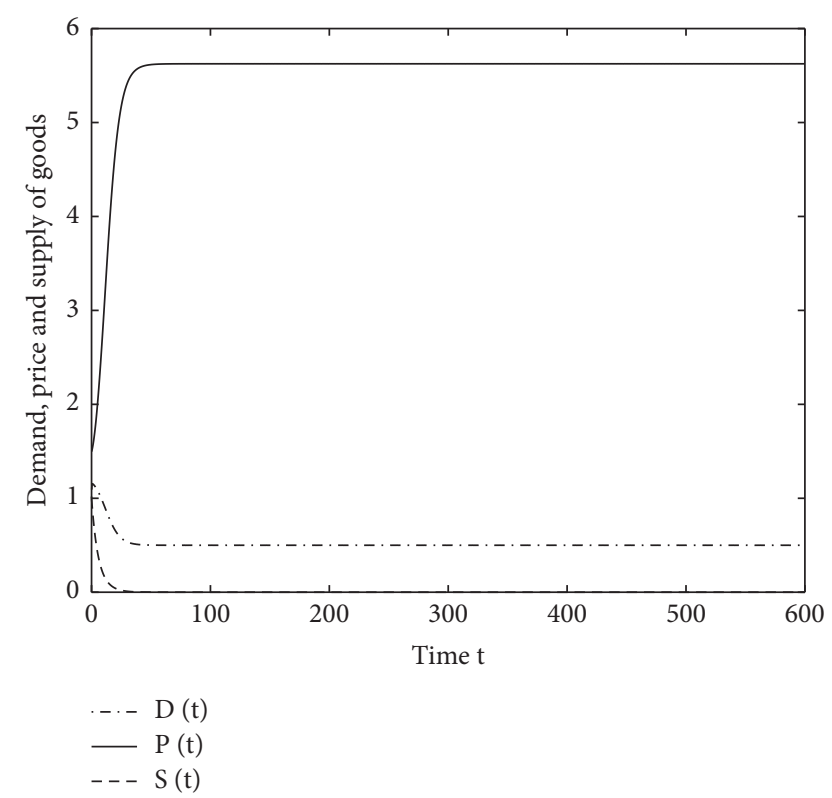

FIGURe 2: Plane phase diagram of the global asymptotic stability at $E_{2}$.

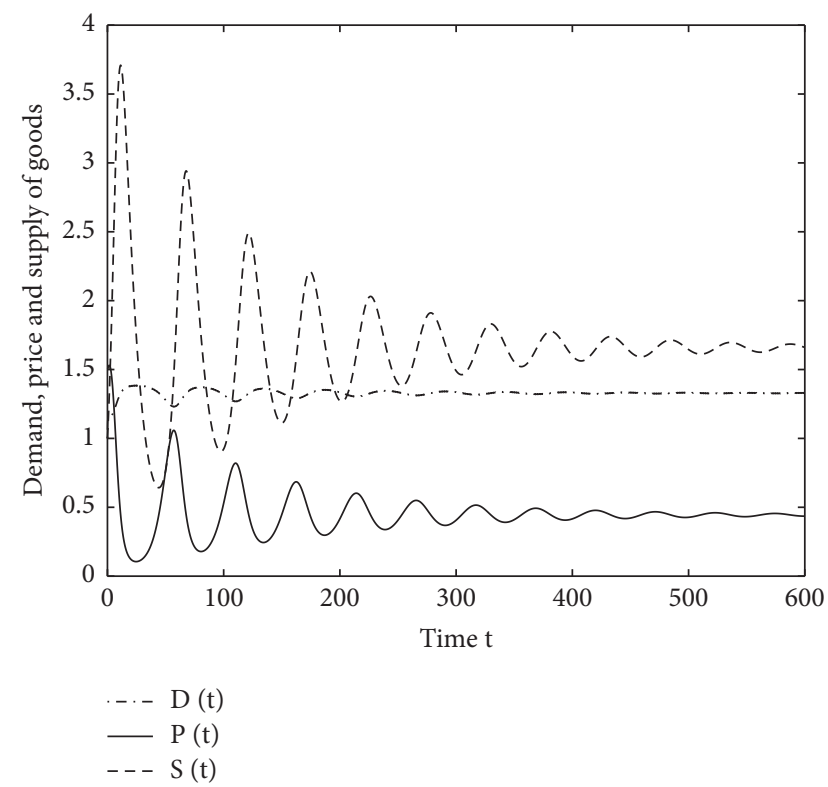

FIGURE 3: Plane phase diagram of the global asymptotic stability at $E_{3}$.

increase of demand. In practice, the decline of price is a gradual process. At the same time, the changes of demand and supply in this process also occur gradually. However, to simplify the problem, this article assumes that production technology cyclically increases, and the demand, price, and supply have changed immediately. Assuming that at $t=k \tau(k=1,2, \ldots)$, after the production technology is improved, the increase in demand is $d_{0}$, the decrease in price is $p_{0}$, and the increase in supply is $s_{0}$. The specific differential dynamic system is as follows.

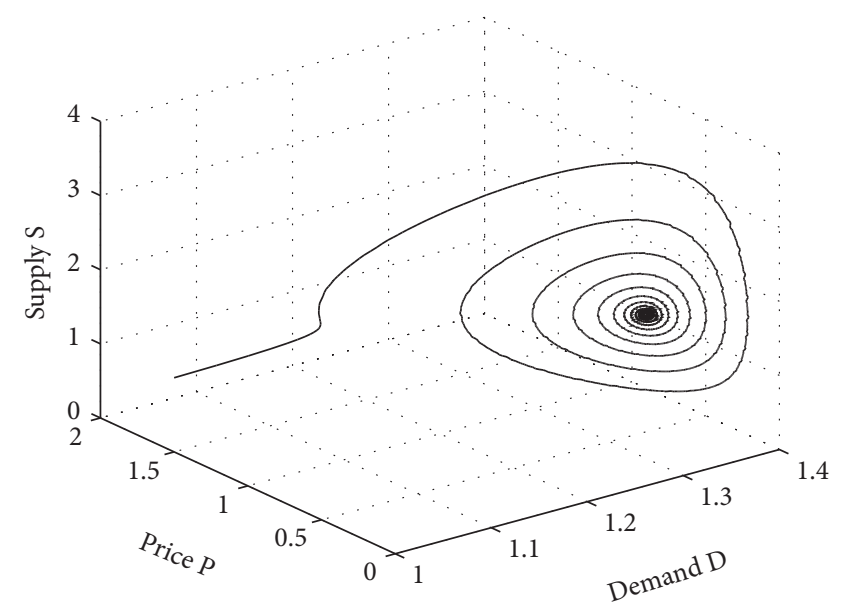

Figure 4: The phase diagram of the global asymptotic stability at $E_{3}$ in space.

$$
\left\{\begin{array}{l}
\frac{\mathrm{d} D}{\mathrm{~d} t}=D\left(e_{1}-k D-\alpha P\right), \\
\left.\frac{\mathrm{d} P}{\mathrm{~d} t}=P\left(-e_{2}+\beta D-\gamma S\right),\right\} \quad t \neq k \tau, \\
\frac{\mathrm{d} S}{\mathrm{~d} t}=S\left(-e_{3}+\frac{\delta P}{\omega+P}\right), \\
D\left(t^{+}\right)=D\left(t^{-}\right)+d_{0}, \\
\left.P\left(t^{+}\right)=P\left(t^{-}\right)-p_{0}, \quad\right\} \\
S\left(t^{+}\right)=S\left(t^{-}\right)+s_{0},
\end{array} \quad t=k \tau,\right.
$$

where $d_{0}>0, p_{0}>0, s_{0}>0, t^{+}=(k \tau)^{+}, t^{-}=(k \tau)^{-}, D\left(t^{+}\right)=$ $\lim _{t \longrightarrow(k \tau)^{+}} D(t), D\left(t^{-}\right)=\lim _{t \longrightarrow(k \tau)^{-}} D(t)$, and so on. $\tau$ is the production technology improvement cycle.

\subsection{Property Analysis of System (32)}

Theorem 8. If $\omega-1 \geq 0$, then all positive solutions of system (32) are still uniformly bounded.

Proof. Still choose the function $V(t)=\beta D(t)+\alpha P(t)$ $+\alpha \gamma / \delta S(t)$ in the proof of Theorem 2 , if $t \neq k \tau(k=1,2, \ldots)$, then

$$
\begin{aligned}
\frac{\mathrm{d} V}{\mathrm{~d} t}+m_{1} V= & \beta\left(e_{1}+m_{1}\right) D-k \beta D^{2}-\alpha\left(e_{2}-m_{1}\right) P \\
& -\frac{\alpha \gamma}{\delta}\left(e_{3}-m_{1}\right) S-\alpha \gamma \frac{\omega-1+P}{\omega+P} P S,
\end{aligned}
$$

where $m_{1}=\min \left\{e_{2}, e_{3}\right\}$. Also, $\omega-1 \geq 0$, so 


$$
\frac{\mathrm{d} V}{\mathrm{~d} t}+m_{1} V \leq \beta\left(e_{1}+m_{1}\right) D-k \beta D^{2} .
$$

Because the function $\beta\left(e_{1}+m_{1}\right) D-k \beta D^{2}$ is bounded, let one of its upper bounds be $m_{2}>0$, and it has

$$
\frac{\mathrm{d} V}{\mathrm{~d} t}+m_{1} V<m_{2} \text {. }
$$

Solving the above differential inequality, we can obtain

$$
V(t) \leq \frac{m_{2}}{m_{1}}+C \exp \left(-m_{1} t\right) .
$$

According to the last three expressions of system (32) and the construction method of the function $V(D, P, S)$, we can get

$$
V\left(t^{+}\right)=V\left(t^{-}\right)+\beta d_{0}-\alpha p_{0}+\frac{\alpha \gamma}{\delta} s_{0} .
$$

In summary,

$$
\begin{cases}V(t) \leq \frac{m_{2}}{m_{1}}+C \exp \left(-m_{1} t\right), & t \neq k \tau, \\ V\left(t^{+}\right)=V\left(t^{-}\right)+\beta d_{0}-\alpha p_{0}+\frac{\alpha \gamma}{\delta} s_{0}, & t=k \tau .\end{cases}
$$

Solve this system of differential inequalities

$$
\begin{aligned}
V(t) \leq & \frac{m_{2}}{m_{1}}+\left[V\left(0^{+}\right)-\frac{m_{2}}{m_{1}}\right] \exp \left(-m_{1} t\right)+\left(\beta d_{0}-\alpha p_{0}+\frac{\alpha \gamma}{\delta} s_{0}\right) \\
& \sum_{i=1}^{k} \exp \left[-m_{1}(t-i \tau)\right]<\frac{m_{2}}{m_{1}}+\left[V\left(0^{+}\right)-\frac{m_{2}}{m_{1}}\right] \exp \left(-m_{1} t\right)+\left(\beta d_{0}+\frac{\alpha \gamma}{\delta} s_{0}\right) \\
& \sum_{i=1}^{k} \exp \left[-m_{1}(t-i \tau)\right]<V\left(0^{+}\right)+\left(\beta d_{0}+\frac{\alpha \gamma}{\delta} s_{0}\right) \\
& {\left[1+\exp \left(-m_{1} \tau\right)+\exp \left(-2 m_{1} \tau\right)+\cdots+\exp (-(k-1) \tau)\right]<V\left(0^{+}\right)+\frac{\left(\beta d_{0}+\alpha \gamma / \delta s_{0}\right)}{1-\exp \left(-m_{1} \tau\right)}, }
\end{aligned}
$$

where $k \tau<t \leq(k+1) \tau(k=1,2, \ldots)$. Take the limit

$$
\lim _{t \rightarrow+\infty} V(t) \leq V\left(0^{+}\right)+\frac{\left(\beta d_{0}+\alpha \gamma / \delta s_{0}\right)}{1-\exp \left(-m_{1} \tau\right)} .
$$

Therefore, $V(t)$ is uniformly bounded. Furthermore, all positive solutions of system (32) are uniformly bounded. The proof is completed.

The conclusion of Theorem 8 shows that there exist three positive numbers $M_{D}, M_{P}, M_{S}$, such that

$$
\lim _{t \longrightarrow+\infty} D(t)<M_{D}, \lim _{t \longrightarrow+\infty} P(t)<M_{P}, \lim _{t \longrightarrow+\infty} S(t)<M_{S} .
$$

Theorem 9. If $\omega-1 \geq 0$, then system (32) is persistent $[18,20,22]$.

Proof. If the initial value of system (32) satisfies $D(0)>0, P(0)>0, S(0)>0$, then its solution $(D(t), P(t), S(t))$ is positive. According to Theorem 8 , if $\omega-1 \geq 0$, then for all $t \in(0,+\infty)$, there exists $M>0$, such that

$$
0<D(t)<M, 0<P(t)<M, 0<S(t)<M,
$$

where $M=\max \left\{M_{D}, M_{P}, M_{S}\right\}$.
Consider the following impulsive differential equation:

$$
\begin{cases}\frac{\mathrm{d} U}{\mathrm{~d} t}=-e_{3} U, & t=k \tau, \\ U\left(t^{+}\right)=U\left(t^{-}\right)+u_{0}, & t \neq k \tau .\end{cases}
$$

According to the conclusion in [23], if the initial value is $U\left(0^{+}\right)=u_{0} / 1-e^{-e_{3} \tau}$, the impulsive differential equation (43) has a global asymptotic stability positive periodic solution

$$
\tilde{U}(t)=\frac{u_{0} \exp \left(-e_{3}(t-k \tau)\right)}{1-\exp \left(-e_{3} \tau\right)} .
$$

That is, for any solution of (43), there are

$$
\begin{aligned}
\lim _{t \longrightarrow+\infty} U(t) & =\widetilde{U}(t) \geq \frac{u_{0} \exp \left(-e_{3} \tau\right)}{1-\exp \left(-e_{3} \tau\right)} \\
& =m_{S}>0 .
\end{aligned}
$$

Thus, there exists $T_{s}>0$; if $t>T_{s}$,

$$
U(t) \geq m_{S} .
$$

If $t \neq k \tau$ and $t>0$, from the third equation of system (32), we can get

$$
\frac{\mathrm{d} S}{\mathrm{~d} t}>-e_{3} S .
$$



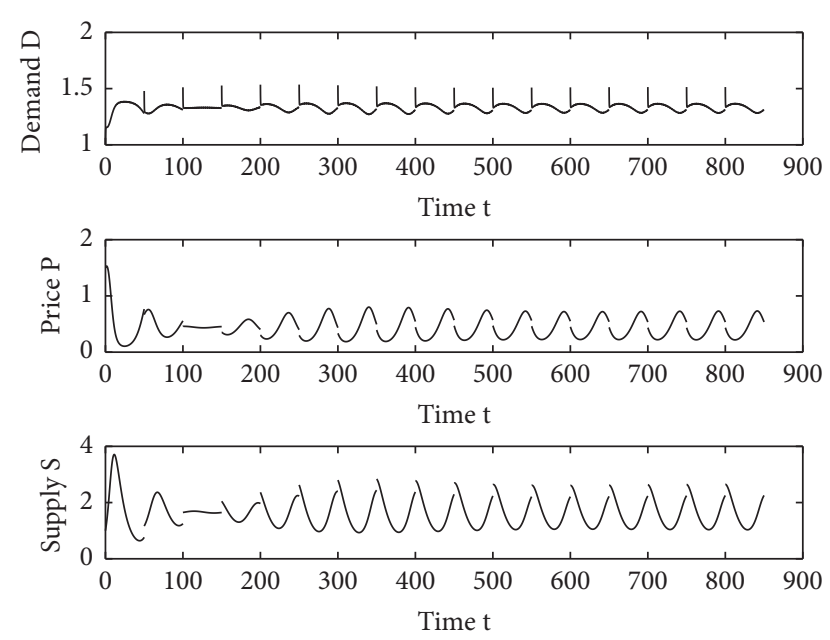

FIGURE 5: Numerical simulation diagram of impulsive differential dynamic system (32).

According to the comparison principle,

$$
M>S(t) \geq U(t) \geq m_{u}>0 .
$$

According to the first and second equations of system (32), we can get

$$
\begin{aligned}
& \frac{\mathrm{d} D}{\mathrm{~d} t}=D\left(e_{1}-k D-\alpha P\right) \geq D(-k M-\alpha M), \\
& \frac{\mathrm{d} P}{\mathrm{~d} t}=P\left(-e_{2}+\beta D-\beta S\right)>P\left(-e_{2}-\beta S\right)>P\left(-e_{2}-\beta M\right) .
\end{aligned}
$$

Using the same method as above, it can be proved that there exist $T_{D}>0, T_{P}>0, m_{D}, m_{P}>0$, so that if $t>T_{D}, t>T_{P}$, we can get

$$
M>D(t) \geq m_{D}>0, M>P(t) \geq m_{P}>0 .
$$

Taking $m=1 / 2 \min \left\{m_{D}, m_{P}, m_{s}\right\}, T=\max \left\{T_{D}, T_{P}, T_{S}\right\}$, if $t>T$, there is

$$
m<D(t), P(t), S(t)<M .
$$

The proof is completed.

The conclusions of Theorems 8 and 9 show that no matter how science and technology improve, with the passage of time, the demand, price, and supply of goods will have upper and lower limits.

Theorem 10. If $\omega-1 \geq 0$, then system (32) has the positive periodic solution.

Proof. According to Theorem 9, if $\omega-1 \geq 0$, $m<D(t)<M(t \in[T,+\infty))$. According to the supremum and infimum principle [24] and the continuity of function $D(t)$, we can get

$$
0<\inf D(t) \leq D(t) \leq \sup D(t), t \in[T,+\infty) .
$$

Define the mapping

$$
[\inf D(t), \sup D(t)] \stackrel{D}{\longrightarrow}(t)=D(t+\tau)[\inf D(t), \sup D(t)] .
$$

According to Brouwer's fixed point theorem [25], there is at least one point $t_{0}(\in[T,+\infty))$ such that $D\left(t_{0}\right)=D\left(t_{0}+\tau\right)$. Therefore, $D(t)$ has at least one positive periodic solution with $\tau$ as the period.

In the same way: $P(t)$ and $S(t)$ all have at least one positive periodic solution with $\tau$ as the period.

In summary, it can be obtained if $\omega-1 \geq 0$, system (32) has a positive periodic solution. The proof is completed.

Theorem 10 shows that demand, price, and supply will fluctuate periodically when production technology is improved periodically.

3.2. Numerical Simulation of System (32). To verify Theorems $8-10$, take parameters $\tau=50, d_{0}=0.2, p_{0}=0.1$, $s_{0}=0.4, k=1,2, \ldots$, and other parameters are the same as those in (29). The numerical simulation of system (32) is shown in Figure 5.

It can be seen from Figure 5 that although the demand will increase instantaneously when the productivity increases, it will return to the normal state immediately. When $t \in[0,300]$, the integral curve of system (32) gradually changes from irregular to regular. When $t \in[300,+\infty)$, $D(T), P(T), S(T)$ appear in the form of cyclic periodic solutions. It is verified that the solution of system (32) is periodic. In addition, when $t \in[0,+\infty), D(t), P(t)$, and $S(t)$ are in a band-shaped region, which verifies that the solution of the system (32) is uniformly bounded and persistence.

\section{Conclusion}

The cobweb model is a traditional model for analyzing economic dynamic changes, which is used to describe the change trend of the interaction of supply, demand, and price. The existing research results are mainly as follows.

(1) The interaction between supply, demand, and price is described by the dynamic relationship between sequences, such as [6-8].

(2) Most of the research results use the external expression relationship among supply, demand, and price to build a cobweb model, which lacks the influence of internal driving force.

(3) A few research results (such as [9]) have constructed a continuous cobweb model, but the focus is on the convergence rate, and the qualitative analysis of the model needs to be more in-depth.

In this paper, a non-linear continuous cobweb economic differential dynamics model is constructed by using the "predator-prey" theory. The specific research results are as follows.

(1) Without the influence of other factors, the relevant conclusions of model (1) show that under certain conditions, the two economic states of self- 
sufficiency and high price but little demand can also be sustained for a long time.

(2) In the state of imbalance, although the supply, price, and demand will continue to fluctuate around the positive equilibrium, the amplitude decreases over time and approaches equilibrium.

(3) Model (32) reveals that when the production technology is cyclically improved, although the supply, price, and demand will fluctuate irregularly in the initial period, all three will change periodically.

This paper mainly studies the dynamic change behavior of the cobweb economic model from the perspective of differential dynamics and gives some properties of the model. These conclusions can provide valuable help for people in the production, sales, or purchase of goods.

\section{Data Availability}

The data used to support the findings of this study are included within the article.

\section{Conflicts of Interest}

The author declares that there are no conflicts of interest regarding the publication of this paper.

\section{Acknowledgments}

This study was supported by the Philosophy and Social Science Program Funds of Heilongjiang Province (19SHE281).

\section{References}

[1] Z. F. Du, Multivariate Statistical Analysis, Tsinghua University Press, Beijing, China, 2016.

[2] X. T. Zhang, Fundamentals of Econometrics, Nankai University Press, Tianjin, China, 2005.

[3] H. Y. Yao, Z. F. Li, and Q. H. Ma, "Maximally linearly independent group of stock returns and separation theorem of two funds," Mathematics in Practice and Theory, vol. 40, no. 17, pp. 14-19, 2010.

[4] Q. Y. Jiang, J. X. Xie, and J. Ye, Mathematical Model, Higher Education Press, Beijing, China, 5th edition, 2018.

[5] D. F. Yang and X. M. Huang, Application and Research of Mathematical Model in Economics, Ocean Press, England, UK, 2015.

[6] X. Bian, A Study on Nonlinear Cobweb Theory and Application, Tianjin University, Tianjin, China, 2005.

[7] Z. L. Huang, "Dynamic analysis on nonlinear and disequilibrium cobweb model," Mathematics in Practice and Theory, vol. 34, no. 3, pp. 40-45, 2004.

[8] W. A. Branch, "Local convergence properties of a cobweb model with rationally heterogeneous expectations," Journal of Economic Dynamics and Control, vol. 27, no. 1, pp. 63-85, 2002.

[9] Y. L. Zhang and Z. C. Yang, "Study of a continuous dynamical multi-products cobweb model with time-delay and its stability," Journal of Southwest University (Natural science edition), vol. 32, no. 11, pp. 69-73, 2010.
[10] Y. P. Zhang, A Course in Microeconomics Theory, China Development Press, Beijing, China, 2005.

[11] C. Castillochavez, Mathematical Models in Population Biology and Epidemiology, Springer, New York, NY, USA, 2012.

[12] W. Gudrun and N. Christian, "Dynamic hybrid modelling: switching between $\mathrm{AB}$ and $\mathrm{SD}$ designs of a predator-prey model," Ecological Modelling, vol. 345, pp. 165-175, 2017.

[13] B.-S. Han and Y.-H. Yang, "On a predator-prey reactiondiffusion model with nonlocal effects," Communications in Nonlinear Science and Numerical Simulation, vol. 46, pp. 49-61, 2017.

[14] F. Zhang, Y. Chen, and J. Li, "Dynamical analysis of a stagestructured predator-prey model with cannibalism," Mathematical Biosciences, vol. 307, pp. 33-41, 2019.

[15] D. B. Gao, "Study on the cobweb economic model with type II functional response," Mathematics in Practice and Theory, vol. 50, no. 15, pp. 133-142, 2020.

[16] H. W. Liu, F. Q. Zhang, and Q. Y. Li, "A prey-predator model in which the carrying capacity of predator depending on prey," Mathematica Applicata, vol. 30, no. 4, pp. 806-813, 2017.

[17] Z. E. Ma, Y. C. Zhou, and C. Z. Li, Qualitative and Stable Methods for Ordinary Differential Equations, Science Press, Beijing, China, 2015.

[18] L. S. Chen, Mathematical Ecology Models and Research Methods, Science Press, Beijing, China, 2017.

[19] C. X. Bu and X. W. Mu, "A note on uniform boundedness of class of time-varying nonlinear systems," Mathematics in Practice and Theory, vol. 34, no. 6, pp. 138-142, 2006.

[20] H. K. Khalil, Nonlinear Systems, Prentice-Hall, New Jersey, NJ, USA, 3rd edition, 2002.

[21] A. Gasull and H. Giacomini, Some Applications of the Extended Bendixson-Dulac Theorem, Springer, New York, NY, USA, 2013.

[22] R. Xu and L. S. Chen, "Persistence and global stability for three-species ratio-dependent predator-prey system with time delays," Journal of Systems Science and Mathematical Sciences, vol. 21, no. 2, pp. 204-212, 2001.

[23] X. Y. Song, H. J. Guo, and X. Y. Shi, Impulsive Differential Equation Theory and its Application, Science Press, Beijing, China, 2011.

[24] A. Browder, Mathematical Analysis: An Introduction, Springer Science \& Business Media, Dordrecht, The Netherlands, 2012.

[25] J. W. Alexander, "On transformations with invariant points," Transactions of the American Mathematical Society, vol. 23, no. 1, pp. 89-95, 1922. 